\title{
Extremely large telescopes and the extremely small sources
}

\author{
J. O. Urama $\dagger$ \\ Department of Physics \& Astronomy, University of Nigeria, Nsukka, Enugu state, Nigeria \\ email: johnsonurama@yahoo.com
}

\begin{abstract}
Neutron stars, containing typically 1.4 solar masses within a diameter of about $15 \mathrm{~km}$, are among the smallest astronomical sources and the densest known form of directly observable matter in the Universe. Many aspects of the neutron star remain poorly understood. Most theoretical models for neutron stars cannot, so far, account for many of the observations, which have been largely made at radio wavelengths. This paper discusses the importance of multiwavelength studies, using large telescopes, to better understand the properties and behaviour of these objects.
\end{abstract}

Keywords. telescopes, stars: neutron, pulsars: general

\section{Introduction}

Neutron stars are one of the final evolutionary stages of stars formed by the collapse of a massive star in a supernova explosion. Containing typically 1.4 solar masses within a diameter of about $15 \mathrm{~km}$, they are also the densest known form of directly-observable matter in the Universe. Although neutron stars have now been recognised in a variety of stellar systems such as X-ray binaries, radio pulsars are by far the most common observable manifestation of the fascinating neutron star. Radio pulsars were discovered as sequences of rapid $(\sim 1 \mathrm{~Hz})$ and regular radio pulses in 1967, by Jocelyn Bell and Anthony Hewish (Hewish et al. 1968). Currently about 1500 pulsars are known and the Galactic population is estimated at around $10^{5}$. The emitted radio pulses allow us to measure directly the rotation of the underlying star. This rotation rate is, in comparison with most other astronomical measurements, exceptionally stable and easy to measure with high accuracy. Glitches and timing noise are perturbations to this regular behaviour which give us a window into the interior of the neutron star.

Much of what is currently known about pulsars, especially the neutron star interior, comes from radio observations. These radio observations continue to provide an excellent probe of the interior of the neutron star, and are unlikely to be surpassed by other techniques. However, less than $0.1 \%$ of the pulsar's spin down energy is emitted at radio wavelengths. Hence radio observations are not very good tools for studying the emission mechanisms and the magnetospheres of pulsars.

\section{Multiwavelength pulsar studies with large optical telescopes}

The fraction of the emitted energy approaches a few percent in the high energy bands and this forms the prime motivation for multiwavelength (especially optical) studies. While nearly all the pulsars have been observed at radio wavelengths, only about $1 \%$ have been detected at other wavelengths - in the optical, X-ray and $\gamma$-ray bands. The

$\dagger$ Presently on leave at the Department of Industrial Physics, Ebonyi State University, Abakaliki, Nigeria 
Crab pulsar is the only one for which IR, optical, and UV radiations have been studied in great details.

These objects are extremely faint optical sources (generally $m_{V}>20$ ). They therefore require bigger and more sophisticated optical telescopes for their study. For example, very recently, Becker et al. (2005) found from a multivalength study of PSR B0628-28 that it emits a huge amount of its spin-down energy in the soft X-ray band and, if confirmed, would make it the first X-ray over-luminous rotation powered pulsar identified among all known radio pulsars. It is not known what could cause such a high X-ray luminosity but extreme re-heating from vortex creep, accretion from the ISM, decay of the pulsar magnetic field, or extreme polar cap heating have been suggested. Observations with larger telescopes have very good potential for detecting pulsed optical emission from a number of other pulsars. These optical emission can yield a wealth of information that is either very difficult or plainly impossible to obtain at other wavelengths. Some of these information, which have been discussed extensively (see, for example, Romani 1998; Urama \& Okeke 1998; Shearer \& Golden 2002) include: luminosity evolution; pulsar emission mechanisms; thermal response to a glitch; study of the binary companions of some of the neutron stars. Furthermore, data from such optical observations using very large telescopes could be combined with those of radio and other high-energy observations for a better understanding of the timing irregularities and the stability of neutron stars.

\section{Conclusion}

The advent of larger telescopes coupled with more sophisticated equipment should herald a period of renewed interest in optical pulsar, and other isolated neutron star, studies as there are many important questions that cannot be answered by observation with most of the existing telescope/instrument systems.

\section{Acknowledgements}

I would like to acknowledge the partial support received from the International Union of Pure and Applied Physics (IUPAP) towards my participation at the IAU Symposium 232. The travel sponsorship came from the International Cooperation Division at the Department of Science and Technology of the South African government.

\section{References}

Becker, W., Jessner, A., Kramer, M., Testa, V., Howaldt, C. 2005, ApJ 633, 367

Hewish, A., Bell, S.J., Pilkington, J.D.H., Scott, P.F., Collins, R.A. 1968, Nature 217, 709

Romani, R.W. 1998 in: D.A.H. Buckley (ed.), Science with SALT - The Southern African Large Telescope Proceedings of the SALT/HET Workshop (Cape Town: SAAO), p. 101

Shearer, A. \& Golden, A. 2002 in: W. Becker, H. Lesch \& J. Trümper (eds.) Neutron Stars, Pulsars and Supernova Remnants Proceedings of the 270. WE-Heraeus Seminar (Germany: MPE Report), vol. 278, p. 44

Urama, J.O. \& Okeke, P.N. 1998, in: D.A.H. Buckley (ed.), Science with SALT - The Southern African Large Telescope Proceedings of the SALT/HET Workshop (Cape Town: SAAO), p. 95 

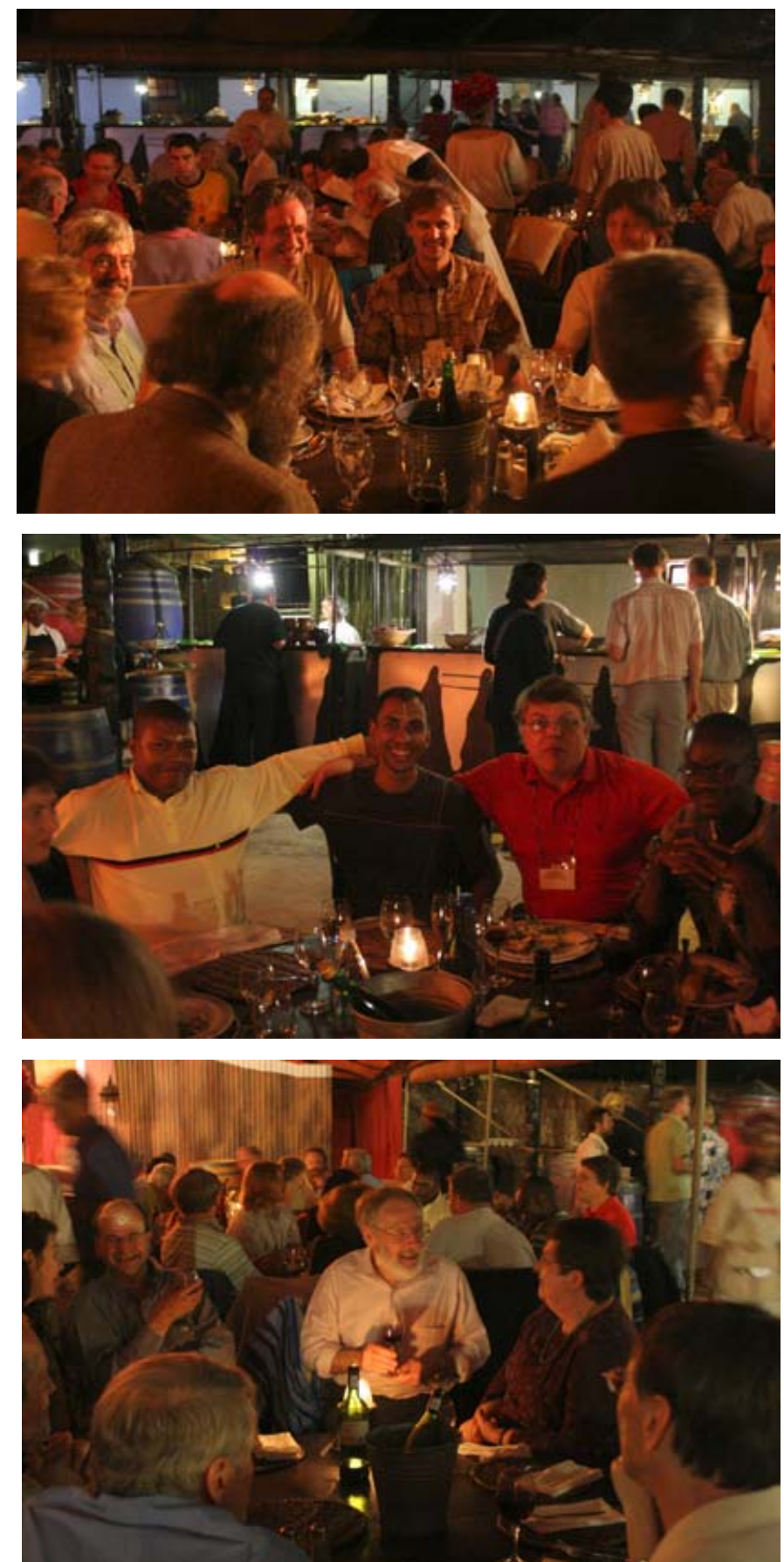

The conference dinner. 